\title{
O que leva homens a se tornar cuidadores informais: um estudo qualitativo
}

\author{
What makes men become informal caregivers: a qualitative study \\ ¿Qué hace con que hombres se vuelvan cuidadores informales?: un estudio cualitativo
}

\author{
Julia Horita Moherdaui' ${ }^{1}$, Carmen Luiza Correa Fernandes ${ }^{2} \mathbb{D}$, Konrad Gutterres Soares ${ }^{3} \mathbb{D}$ \\ ${ }^{1}$ Universidade do Estado do Rio de Janeiro (UERJ). Rio de Janeiro, RJ, Brasil. \\ ${ }^{2}$ Grupo Hospitalar Conceição (GHC). Porto Alegre, RS, Brasil. \\ ${ }^{3}$ Instituto Municipal de Estratégia de Saúde da Família (IMESF). Porto Alegre, RS, Brasil.
}

\section{Resumo}

Introdução: O aumento da expectativa de vida tem levado a um aumento do número de pessoas que necessitam de cuidado, sendo a maior parte dele exercido por cuidadores informais e do sexo feminino. Entretanto, diversos estudos têm apontado um aumento no número de cuidadores masculinos. Neste contexto, este estudo pretende elencar motivações que levam homens a serem cuidadores informais. Métodos: Estudo qualitativo com entrevistas semiestruturadas, questionários sociodemográficos e questionários de Zarit em dez cuidadores homens inscritos no programa de atenção domiciliar de duas unidades de saúde de Porto Alegre. Resultados e Discussão: As motivações identificadas na prestação do cuidado foram a obrigação e a reciprocidade. A obrigação foi citada por todos os cuidadores e a falta de rede de apoio pareceu reforçá-la. A reciprocidade foi mencionada em quatro entrevistas e possuía maior correlação com o grau de parentesco "filho". Estudos apontam que a motivação "obrigação" parece se correlacionar com maiores índices de sobrecarga, depressão e ansiedade quando comparado a um cuidado motivado pela reciprocidade. Na escala de Zarit, nenhum cuidador apresentou índice de sobrecarga severa, o que pode ter relação com uma maior procura por suporte social. Conclusão: Nota-se como é amplo e desconhecido o tema da relação da masculinidade com o papel de cuidador. Contudo, observou-se correlação entre o gênero e as motivações como obrigação e reciprocidade na prestação do cuidado, e insinua-se uma maior sobrecarga quando obrigação é o principal fator motivador.

Palavras-chave: Cuidadores; Homens; Gênero e Saúde; Masculinidade; Motivação

Como citar: Moherdaui JH, Fernandes CLC, Soares KG. O que leva homens a se tornar cuidadores informais: um estudo qualitativo. Rev Bras Med Fam Comunidade. 2019;14(41):1907. https://doi.org/10.5712/rbmfc14(41)1907
Autor correspondente: Julia Horita Moherdaui. E-mail: juliahorita@gmail.com Fonte de financiamento: declaram não haver.

Parecer CEP: não se aplica.

Procedência e revisão por pares: revisado por pares. Recebido em: 16/01/2019. Aprovado em: 05/08/2019. 


\begin{abstract}
Introduction: The increase in life expectancy has led to a larger number of people who need care, which is mostly provided by informal and female caregivers. However, studies have shown a growth in the number of male caregivers. In this context, this study intends to observe motivations that make men become informal caregivers. Methods: Qualitative study with semi-structured interviews, sociodemographic questionnaires and Zarit questionnaires answered by ten male caregivers registered in the home care program of two primary health care clinics in Porto Alegre. Results and Discussion: The motivations identified in the caregiving process were obligation and reciprocity. Obligation was mentioned by all of the caregivers and the lack of a support network seemed to reinforce it. Reciprocity was mentioned in four interviews and was mostly correlated to sons who took care of their parents. Studies show that the motivation "obligation" seems to be related to higher levels of caregiver burden, depression and anxiety when compared to the motivation "reciprocity". In the Zarit scale, there weren't caregivers with severe burden, which can be related to a greater demand for social/professional support. Conclusion: The theme of the relation between caregiving and masculinity is vast and unknown. However, we could observe a correlation between gender and caregiving motivations as obligation and reciprocity, and it is suggested that the motivation "obligation" can be related to a more severe burden.
\end{abstract}

Keywords: Caregivers; Men; Gender and Health; Masculinity; Motivation

\title{
Resumen
}

Introducción: El incremento de la expectativa de vida ha llevado a un aumento del número de personas que necesitan cuidado, en gran parte ejercido por cuidadores informales del sexo femenino. Entretanto, muchos estudios indican un aumento en el número de cuidadores hombres. En ese contexto, este trabajo desea enumerar motivaciones que llevan hombres a ser cuidadores informales. Métodos: Estudio cualitativo con entrevistas semi estructuradas, cuestionarios sociodemográficos y cuestionarios Zarit en diez cuidadores hombres inscritos en el programa de atención domiciliaria de dos unidades de salud de Porto Alegre. Resultados y Discusión: Las motivaciones identificadas en la prestación de cuidado fueron la obligación y la reciprocidad. La obligación fue citada por todos los cuidadores y la falta de red de apoyo pareció reforzarla. La reciprocidad fue mencionada en cuatro entrevistas y tenía más correlación con el parentesco "hijo". Estudios apuntan que la motivación "obligación" parece correlacionarse con índices más altos de sobrecarga, depresión y ansiedad cuando se compara a un cuidado motivado por la reciprocidad. En la escala de Zarit, ningún cuidador tuvo índice de sobrecarga severa, lo que puede tener relación con una más alta búsqueda por suporte social. Conclusión: Se observa cómo es amplio y desconocido el tema de la relación de la masculinidad con el papel de cuidador. Sin embargo, parece existir una correlación entre el género y las motivaciones en la prestación de cuidado, y se sugirie que haya relación entre sobrecarga y la motivación "obligación".

Palabras clave: Cuidadores; Hombres; Género y Salud; Masculinidad; Motivación

\section{Introdução}

Vivemos num contexto de notável aumento da expectativa de vida. Nos Estados Unidos da América (EUA), há grande crescimento da população idosa, com consequente aumento da prevalência de múltiplas doenças crônicas. ${ }^{1}$ Em 2010, nos EUA, as pessoas com limitações do autocuidado eram 10 milhões, e estima-se que esse número venha a crescer para 15 milhões até o ano $2020 .{ }^{2}$ No Brasil, existe a mesma tendência: aumento da proporção de idosos entre 1999 e 2009, com ainda maior crescimento da população de maiores de 80 anos, sendo essa uma população a quem é mais dispendioso prestar cuidado, tanto em termos de recursos financeiros quanto humanos. ${ }^{3}$

Nesse contexto, a projeção do aumento da necessidade de cuidadores torna-se essencial. A maior parte do cuidado é hoje exercida por cuidadores informais. ${ }^{4}$ Definimos como cuidador informal o indivíduo não remunerado que presta cuidados à pessoa em seu domicílio, independentemente do vínculo familiar. ${ }^{5}$ As preocupações com essa pessoa, frequentemente negligenciada pelos serviços de saúde, devem-se a conhecimentos estabelecidos sobre a influência emocional e física do ato de cuidar. O chamado "estresse do cuidador" é classicamente definido como "tensão ou carga suportada pela pessoa que cuida de um idoso, de um doente crônico, ou de uma pessoa incapacitada, (...) sendo uma resposta multidimensional a estressores físicos, emocionais e financeiros, associada à experiência do cuidador". 6 
A sobrecarga do cuidador é tema relevante para saúde e é responsabilidade do clínico o seu diagnóstico. ${ }^{4}$ Para auxiliar no diagnóstico e classificação de severidade desta sobrecarga existem instrumentos, sendo o Zarit Burden Interview o mais utilizado. Esse questionário aborda diversas áreas relacionadas à sobrecarga do cuidador, incluindo sua saúde, bem-estar psicológico e financeiro, vida social e sua relação com a pessoa que recebe cuidado. ${ }^{7}$ Trata-se de um tema bastante discutido, mas ainda sabe-se pouco sobre suas relações com os fatores que podem afetá-lo, como estratégia de cuidado, estado marital, estado empregatício, parentesco com a pessoa que recebe cuidado, suporte institucional, idade e gênero. ${ }^{8}$

Gênero é uma construção histórica e social que diferencia indivíduos de uma sociedade na impressão de comportamentos, normas e relações de poder, a partir de distinções percebidas entre os sexos. Envolve necessariamente aspectos relacionais entre masculinidades e feminilidades. ${ }^{9}$ Historicamente, não se tratou de um campo examinado como determinante social em saúde, sendo apenas mais recentemente observado para além das diferenças relacionadas aos sexos biológicos. ${ }^{10}$

Nas culturas ocidentais, o cuidado se associa ao gênero feminino: é mais frequente que mulheres assumam o papel de cuidadoras, que executem mais tarefas quando cumprem esses papéis e que passem mais tempo cuidando que homens. ${ }^{11}$ Por mais que o cônjuge venha a se tornar o cuidador na maioria dos casos, é menos provável que esposas recebam cuidado de seus esposos do que o contrário. ${ }^{2}$ Isso, na verdade, não ocorre apenas no que diz respeito ao "cuidar do outro", mas também ao "cuidar de si". Courtenay ${ }^{10}$ discute como faz parte da construção das masculinidades evitar o cuidado em saúde, de maneira a manter a prescrição social de serem robustos, fortes, independentes.

Apesar disso, é perceptível um aumento da proporção de homens exercendo o papel de cuidador informal de seus familiares dentro do contexto da atenção primária em saúde. Em países do Reino Unido, os homens cuidadores já são maioria quando se examina o grupo de cuidadores com mais de 75 anos. ${ }^{12}$ Essa mudança do perfil de gênero de cuidadores se dá dentro num contexto de crise do sistema informal de cuidado. Cajado-Mejía \& Ruiz-Arias ${ }^{8}$ descrevem tal crise observando que mulheres se fazem mais presentes no mercado de trabalho enquanto homens não se responsabilizam pelo cuidado na mesma proporção.

O mesmo artigo afirma que ser do gênero feminino é um dos fatores que mais aumenta a sobrecarga do cuidador, o que é consistente com a literatura em geral. Isso é relacionado a uma negligência no autocuidado em prol da atenção ao cuidado de outrem, tendo em vista a construção social do papel de gênero feminino. ${ }^{8}$ Isso é agravado por fatores como o baixo nível educacional, o diagnóstico de depressão, o isolamento social, coabitar com a pessoa que demanda cuidado, o número de horas dedicadas ao cuidado e a sensação de falta de escolha em tornar-se cuidador(a). ${ }^{4}$

Este estudo tem o objetivo de identificar motivações que levam homens a serem cuidadores informais e levantar hipóteses sobre a influência de tais motivações sobre o estresse do cuidador. Justifica-se pela mudança global do perfil de gênero desses cuidadores informais, que anteriormente contava com uma enorme preponderância feminina. 


\section{Método}

Foi escolhida a metodologia qualitativa, associada à metodologia quantitativa auxiliar. Utilizamos entrevistas semiestruturadas como técnica principal de coleta de dados e questionários sociodemográficos como técnica auxiliar, além do uso do questionário de Zarit Burden Interview, validado no Brasil, ${ }^{13}$ para avaliação individual de estresse do cuidador: nenhuma ou pequena sobrecarga (0-20 pontos); sobrecarga leve a moderada (21-40 pontos); sobrecarga moderada a severa (41-60 pontos); e sobrecarga severa (61-88 pontos).

A população estudada é formada de homens cuidadores de idosos dependentes de cuidado indicados por profissionais médicos de duas unidades de Atenção Primária à Saúde da zona norte de Porto Alegre. Ambas as unidades atuam no modelo de Estratégia Saúde da Família, fazem visita domiciliar, são instituições públicas que atendem pelo Sistema Único de Saúde e apresentam cobertura universal. Foi selecionada uma amostra de conveniência de dez usuários, sendo cinco de cada unidade de saúde.

Os critérios de inclusão para o estudo foram: ser cuidador do gênero masculino do Programa de Atenção Domiciliar das unidades de saúde participantes, não receber remuneração pelo cuidado e compreender e assinar um Termo de Consentimento Livre e Esclarecido (TCLE). Os critérios de exclusão foram: ser cuidador profissional ou recusar-se a participar do estudo. Todos os cuidadores tinham acesso ao serviço de saúde prestado pelas unidades em questão e foram orientados que em caso de não participação no estudo isso não alteraria a assistência prestada.

As entrevistas foram realizadas nas unidades de saúde (três entrevistas) e no domicílio dos entrevistados (sete entrevistas), conforme sua preferência. Os selecionados foram submetidos inicialmente a questionário sociodemográfico estruturado para caracterização da amostra. Foram incluídas questões sobre idade, estado civil, escolaridade, cor, orientação sexual, renda familiar, situação empregatícia, parentesco com a pessoa que recebe cuidado e horas diárias dedicadas ao cuidado. Depois, foram realizadas entrevistas semiestruturadas subdivididas nos seguintes tópicos: "motivações e sentimentos em relação ao cuidado" e "gênero e cuidado", numa lista de perguntas adaptadas de del Río-Lozano et al. ${ }^{14}$ (Quadro 1). Por fim, foi aplicada a versão brasileira da Zarit Burden Interview ${ }^{13}$ para avaliação do estresse do cuidador.

As entrevistas foram realizadas pelo segundo autor, que é do gênero masculino, para eliminar possíveis vieses de informação relacionados a uma diferença de gêneros entre entrevistado e entrevistador. Foram posteriormente transcritas literalmente pelos dois autores e analisadas a partir de sua leitura exaustiva. A análise dos dados correspondeu aos seguintes procedimentos: categorização sistemática do conteúdo, tabulação dos temas das falas, identificação de semelhanças nos discursos, correlação dos temas com os referenciais teóricos utilizados e interpretação e análise crítica do conteúdo, contando com a participação dos dois autores em todas as etapas. Com isso, identificamos duas categorias principais: obrigação e reciprocidade. O conteúdo das entrevistas somou cerca de 5 horas, sendo a média de 28 minutos por entrevista. Foi utilizado o programa NVivo 11 como ferramenta auxiliar.

A pesquisa foi aprovada pelo Comitê de Ética em Pesquisa do Hospital Nossa Senhora da Conceição - GHC (parecer número 2.171.173). Este estudo não recebeu financiamento para sua realização. 
Quadro 1. Perguntas da entrevista semiestruturada.

\begin{tabular}{|l|}
\hline Motivações/Sentimentos: \\
\hline Como você se tornou um cuidador? \\
Oor quê você é o cuidador principal? \\
Como as responsabilidades familiares são compartilhadas em casa? \\
Como você se descreveria como cuidador? \\
Quais são os principais sentimentos que ser um cuidador lhe traz? \\
Você acha que é um cuidador competente? Sim, não e por quê? \\
\hline Gênero: \\
\hline O que é ser homem/mulher para você? \\
Por quê você acha que na maioria das vezes são mulheres as cuidadoras? \\
Por quê você acha que homens se tornam cuidadores? \\
Como você acha que cuidar afeta a sua masculinidade? \\
Como você acha que o seu círculo social pensa sobre o seu papel de cuidador? \\
\hline
\end{tabular}

Fonte: adaptado de del Río-Lozano et al. ${ }^{14}$

\section{Resultados e Discussão}

\section{Caracterização Sociodemográfica}

Conforme a Tabela 1, seis dos entrevistados tinham mais de 60 anos. $O$ cuidador mais jovem tinha 39 anos e o mais idoso 73 anos. Oito eram casados. Todos os entrevistados (dez) se consideravam homens heterossexuais. Sete deles se declararam como brancos e três como negros. Quando questionados sobre sua escolaridade, cinco dos cuidadores estavam cursando ou já tinham cursado ensino superior, enquanto três não tinham completado o ensino fundamental. Em relação à renda, cinco possuíam renda familiar entre 4 e 8 salários mínimos, outros três entre 2 e 4 salários mínimos, outros dois com renda de até 2 salários mínimos. Quando a variável foi a presença de vínculo empregatício, encontramos quatro sujeitos aposentados, três atuando como autônomos, dois assalariados e um desempregado.

Tabela 1. Características sociodemográficas dos sujeitos.

\begin{tabular}{lccccccc}
\hline & Idade & Cuidado (horas) & Vínculo & $\begin{array}{c}\text { Situação } \\
\text { Empregatícia }\end{array}$ & Renda & Rede de Apoio & Zarit \\
\hline 1 & 61 & 12 & marido & autônomo & Até $\mathrm{R} \$ 1.760$ & Não & 22 \\
2 & 44 & 7 & filho & autônomo & $\mathrm{R} \$ 1.760-3.720$ & Sim & 13 \\
3 & 72 & 9 & marido & aposentado & $\mathrm{R} \$ 1.760-3.720$ & Sim & 28 \\
4 & 39 & 4 & filho & assalariado & $\mathrm{R} \$ 3.720-8.800$ & Não & 9 \\
5 & 64 & 18 & marido & autônomo & $\mathrm{R} \$ 3.720-8.800$ & Não & 33 \\
6 & 60 & 24 & filho & aposentado & $\mathrm{R} \$ 3.720-8.800$ & Não & 40 \\
7 & 73 & 24 & marido & aposentado & Até $\mathrm{R} \$ 1.760$ & Não & 17 \\
8 & 57 & 4 & filho & desempregado & $\mathrm{R} \$ 1.760-3.720$ & Sim & 13 \\
9 & 50 & 4 & filho & assalariado & $\mathrm{R} \$ 3.720-8.800$ & Sim & 30 \\
10 & 72 & 24 & marido & aposentado & $\mathrm{R} \$ 3.720-8.800$ & Não & 42 \\
\hline
\end{tabular}


Cinco dos cuidadores cuidavam de suas esposas, os outros cinco de suas mães. Quanto ao tempo de cuidado, seis relataram um cuidado de menos de 12 horas por dia e quatro relataram um cuidado de mais de 12 horas. A presença de rede de apoio, formada por outros familiares que participem do cuidado, foi identificada em seis dos entrevistados.

\section{Motivação}

As motivações que levam ao cuidado têm sido pouco exploradas na literatura. Estudos anteriores identificaram que as seguintes motivações: gratidão e sentimento de reciprocidade, sentimento de responsabilidade ou dever e afeto ou proximidade. ${ }^{15}$ Sharma et al. ${ }^{16}$ descrevem como fatores de motivação em cuidadoras do gênero feminino a conexão emocional e social com os seus familiares, bem como o seu senso de família e obrigação. As mulheres parecem estar mais preocupadas com o bem-estar emocional das pessoas de quem elas cuidam e também apresentam um senso maior de responsabilidade para com o enfermo. O altruísmo e a doação pessoal caracterizam o cuidado feito por mulheres. Contudo, estudos com cuidadores masculinos sugerem que o cuidado por homens também é conduzido por um senso semelhante de carinho, compromisso e obrigação familiar. ${ }^{16}$

Certos estudos demonstram que cuidadores com uma motivação de carga emocional negativa para o cuidado apresentam maior tendência a sobrecarga, comportamento abusivo, ansiedade e maior chance de institucionalização do enfermo. ${ }^{17,18}$

Nota-se que existem motivações diferentes que possivelmente são influenciadas pelo gênero e que a sua identificação possibilitaria correlação com desfechos negativos. Buscamos, portanto, identificar se esses motivos estavam presentes nos homens sujeitos deste estudo. Pudemos identificar neles principalmente duas categorias de motivações nas entrevistas realizadas: a obrigação e a reciprocidade.

\section{Obrigação como Determinante da Motivação:}

Um dos sentimentos determinantes na prestação do cuidado entre nossos sujeitos foi a sensação de obrigação. A percepção do dever familiar chamou a atenção em diversos relatos, inclusive ressaltando a não importância do gênero para essa definição como demonstra o caso do sujeito 2, filho que cuida há muitos anos da mãe que tem doença cardíaca:

"Filho único, a obrigação é para mim. Independentemente de ser homem ou mulher, se eu fosse sozinho, filho único, tanto faz homem ou mulher. Tem que cuidar da mãe até o fim."

Relatos semelhantes apareceram em outras entrevistas. O vínculo familiar de filho ou marido parece cristalizar-se como um dos determinantes da motivação de obrigação social e moral na prestação do cuidado. O sujeito 1 reforça a importância do papel de marido como determinante na obrigação (ainda que mesclado à reciprocidade no discurso):

"Eu sou marido, né? Tenho obrigação com a esposa, na minha visão. Ela dedicou tantos anos da vida dela para mim, cuidando de mim. É justo que eu cuide dela." 
O entrevistado 6 reforça também a relevância do papel de filho:

"Sou filho único, meu pai e minha mãe moram do lado de casa e é aquilo que eu te disse, sou cuidador por opção. Podia ter colocado desde o início em algum lugar, mas eu sinto que tenho essa obrigação como filho, por tudo que ela fez para mim."

Nas entrevistas de alguns cuidadores surgiu a característica marcante de falta de rede de apoio, o que nesses casos se somava aos relatos da sensação de obrigação. Esses achados são exemplificados pelo caso do sujeito 5 , que cuida de esposa com uma doença neurodegenerativa:

"Por necessidade, por não ter alguém para fazer esse trabalho, e por uma obrigação de marido. Então foi por uma obrigação familiar e de marido."

As respostas de outras duas entrevistas reforçam essa impressão (entrevistas 3 e 7, respectivamente):

"Necessidade, não tinha quem cuidasse. Tinha que ser eu. Porque não tem quem cuide. Então foi eu mesmo." "Porque não tinha outra pessoa. Tinha só eu para cuidar dela."

Todos os sujeitos apresentaram em algum momento de suas falas pensamentos ou ideias de obrigação na prestação do cuidado. A importância dessa identificação se correlaciona a achados de outros estudos que apontam que cuidadores familiares motivados pela obrigação tendem a maiores índices de sobrecarga, depressão e ansiedade do que quando comparados aos motivados pela reciprocidade. ${ }^{19}$

\section{Reciprocidade como Determinante da Motivação}

Dentre as motivações na prestação do cuidado, foi identificado o sentimento de reciprocidade em quatro sujeitos. Dwyer et al. ${ }^{20}$ relacionam o conceito de reciprocidade à compensação por alguma ação ou serviço, fornecendo ainda os conceitos de reciprocidade específica, que é aquela imediata, e reciprocidade generalizada, aquela em que as trocas se equilibram ao longo de toda a vida - esta bem mais notável em relações familiares como as estudadas aqui. Essa motivação foi referida por cuidadores que possuíam o parentesco "filho", sendo encontrada em três dos cinco entrevistados que pertenciam a essa categoria. É o caso do entrevistado 8, que assumiu o cuidado dos pais idosos. O pai faleceu em 2015, mas ele permanece cuidando da mãe. Possui suporte familiar e uma irmã que o auxilia, mas optou por assumir o papel de cuidador principal pelo sentimento de reciprocidade:

"Quando eu nasci, ela que me cuidou. Agora mudou, agora eu que tenho que cuidar dela. Eu tenho que retribuir tudo que ela fez por mim, é isso que eu penso." 
Ele inclusive reforça em seu discurso que apenas exerce esse papel devido ao vínculo familiar, mesclando a isso um sentimento de obrigação social - demonstrando dessa forma como as motivações se misturam:

"Eu fui pelo sentimento, alguém tem que cuidar e eu me ofereci. É a minha mãe. Mas se tu diz ir cuidar de outra pessoa, outra família, aí já não, porque não é a minha profissão. Eu estou cuidando porque é a minha família."

Outro filho cuidador é o entrevistado 9 , um sujeito de 50 anos, que cuida da mãe com fratura de fêmur e limitação funcional. Ele apresenta uma narrativa semelhante em que se nota a ambivalência de sentimentos na prestação do cuidado:

"Foi automático, eu sou filho dela e moro com ela. Como eu moro com os pais, fui obrigado a moralmente e afetuosamente a cuidar dela." "É uma retribuição em decorrência do sentimento de afeto familiar."

A retribuição do cuidado por parte dos filhos para com seus pais surge como um fator relevante nos cuidadores masculinos. Estudos com mulheres cuidadoras também identificaram este sentimento e apontam possível relação desta motivação com uma maior satisfação na prestação do cuidado, melhor autoestima e maior predisposição a procurar suporte social. ${ }^{21}$

A variável reciprocidade não foi percebida com a mesma intensidade quando o cuidador era o marido. No caso do sujeito 1, um senhor de 61 anos que cuida da esposa com uma doença neurodegenerativa e possui fraca rede de apoio, ela parece ser influenciada pela convivência de muitos anos:

"Talvez seja pela nossa vivência de vida inteira, mais de 40 anos juntos. Eu acho que se fosse eu que estivesse doente ela cuidaria de mim."

O entrevistado 5 também apresentou um relato semelhante:

"Cuidador principal eu diria por uma exigência e necessidade dela, e por termos essa convivência de tantos anos, tantos anos que a gente se conhece, sabendo das necessidades que um tem com o outro. E pela confiança."

Portanto, pode-se inferir a hipótese de que o sentimento de retribuição possui um papel importante na motivação de alguns cuidadores e tende a ter uma maior importância quando o grau de parentesco é "filho". A reciprocidade parece perder importância à medida que a rede de apoio se enfraquece, pois nesse contexto o sentimento de obrigação surge provavelmente como um fator negativo na percepção do cuidador. 


\section{Sobrecarga do cuidador}

A escala de Zarit foi aplicada a todos os sujeitos, sendo que quatro apresentaram sobrecarga pequena ou nenhuma, cinco apresentaram sobrecarga leve a moderada e um moderada a severa. Nenhum sujeito apresentou sobrecarga severa.

Essa ferramenta trouxe dados quantitativos. Como não houve cálculo estatístico e de amostra, as inferências sobre esses resultados não possuem validade externa. Entretanto, a escala possui validade individual, ou seja, para análise de cada cuidador. Encontramos possíveis correlações com as informações coletadas e formulamos algumas hipóteses descritas a seguir.

Dentre os entrevistados, os que tinham maior número de horas prestando cuidado foram os que obtiveram uma pontuação maior no questionário de Zarit, ou seja, são os que sofrem com maior estresse do cuidador. Percebe-se também que entre os cuidadores que referiram a obrigação como motivação essa sobrecarga é maior, especialmente naqueles que acreditavam na irreversibilidade da situação, condizendo com o que afirmam Schrank et al. ${ }^{18}$ ao apresentar a esperança como fator protetor contra a sobrecarga. Os sujeitos que possuem renda mais alta tiveram níveis de estresse mais alto, o que pode significar que o cuidar, nesses casos, cerceie possibilidades: talvez se não fossem cuidadores usufruissem de outra maneira de seus altos ganhos. Sob outra perspectiva, o estudo de Lee \& Tang ${ }^{22}$ apontou que homens sentem-se pressionados financeiramente, o que demonstra a influência do dinheiro na saúde do cuidador.

Outro aspecto relevante foi a ausência de cuidadores masculinos com índices de sobrecarga severa em nosso serviço. Isso contrasta com os achados de Milligan \& Morbey, ${ }^{23}$ que discutem a recusa masculina a buscar auxílio de serviços de saúde antes que se atinja um ponto de máximo de crise. Essas mesmas autoras apresentam uma dificuldade desses homens de se perceberem como cuidadores ou mesmo de se verem sob estresse, o que pode ter trazido um viés de informação na aplicação do questionário Zarit. Além disso, Schrank et al. ${ }^{18}$ sugerem que o acesso a serviços de suporte a cuidadores e identificação de cuidadores sob estresse funciona como um indicador de qualidade do cuidado paliativo. Dessa forma, pode ser que o acesso às unidades de saúde estudadas seja considerado um fator de proteção destes cuidadores.

Chama atenção que o sujeito com a mais alta sobrecarga (42 pontos) segundo o questionário Zarit apresenta em seu discurso uma forte ambiguidade no que tange à motivação para o cuidado. Ao mesmo tempo em que afirma cuidar de sua esposa por opção, "causa humanista", traz à luz em vários momentos a necessidade como um determinante de suas ações. Por exemplo:

"Eu me considero um soldado. Fui militar muitos anos, então uma das metas do militar é não ver sacrifício nas coisas. Tem que fazer, tem que fazer."

É interessante perceber também como há uma visão da masculinidade hegemônica, que representa uma rejeição ao feminino, segundo Courtenay, ${ }^{10}$ através da simbolização do militar como estereótipo de gênero masculino: forte, robusto, independente e confiável. 


\section{Fortalezas e limitações do estudo}

Este estudo apresenta fortalezas e limitações características do método. Segundo Dilthey, os pesquisadores qualitativos "explicam a natureza, compreendem a vida mental" (como citado por Gunther, p. $202^{24}$ ). A pesquisa qualitativa tem a vantagem de trabalhar com reflexões e processos, de forma que mesmo informações de caráter objetivo adquirem sentido subjetivo. ${ }^{24}$ Assim, este método é composto por um espectro de métodos e técnicas adaptável ao objeto de estudo, muito menos controlável, ou seja, sujeita a variáveis imprevistas - o que pode ser visto como um dificultador ou uma forma de expandir o conhecimento acerca do tema. Por isso, esta pesquisa tem a vantagem de poder servir como base para novas investigações, tanto quantitativas quanto qualitativas, acerca do tema trabalhado. Existem porém limitações, como a validade externa diminuta, dadas as características particulares da pequena população com que trabalhamos.

\section{Conclusões}

Com a expectativa de identificar algumas das motivações que levam à definição de homens como cuidadores informais, dada a mudança global do perfil de gênero de cuidadores informais - da enorme preponderância feminina para um aumento notável de cuidadores do gênero masculino - além de identificar o impacto do papel social de gênero no estresse decorrente do cuidado, este trabalho pretende participar de um movimento de pesquisa das masculinidades que hoje está em crescimento. Dessa forma, acreditamos que o conhecimento adquirido proporcionará aos profissionais a possibilidade de se preparar para atender de forma mais adequada seus usuários que cumprem o papel de cuidadores, colaborando para o fortalecimento de sua rede de apoio e a melhora de sua qualidade de vida.

Neste trabalho, identificamos duas principais motivações dos homens do nosso território para ser cuidadores, e ambas já haviam sido identificadas na literatura. Chama a atenção, contudo, que elas possuem aparentemente correlação com desfechos distintos. Levanta-se a hipótese de que o sentimento de obrigação esteja mais associado com maiores índices de sobrecarga, como observado neste estudo, ou mesmo de depressão e ansiedade. Enquanto isso, a reciprocidade pode figurar como um fator protetor, com maior satisfação na prestação do cuidado, melhor autoestima e maior predisposição a procurar suporte social.

Com este trabalho, nota-se como é amplo e desconhecido o tema da relação da masculinidade com o papel de cuidador. O estudo deve estimular e abrir portas para o aprofundamento do conhecimento acerca das relações entre gênero, motivação e sobrecarga. Algumas das perspectivas de investigações que esta pesquisa levanta são: diferenças de gênero na motivação do cuidado, desfechos relacionados com as motivações no cuidado, diferenças de gênero na prestação do cuidado, hipóteses sobre fatores protetores em relação à sobrecarga do cuidado e estratégias para lidar com a sobrecarga desses cuidadores. ${ }^{25}$

\section{Contribuição dos autores}

Concepção e/ou delineamento do estudo: JHM, KGS, CLCF. Aquisição, análise ou interpretação dos dados: JHM, KGS. Redação preliminar: KGS. Revisão crítica da versão preliminar: JHM, KGS. Todos os autores aprovaram a versão final e concordaram com prestar contas sobre todos os aspectos do trabalho. 


\title{
Conflito de interesses
}

\author{
Declaram não haver.
}

\section{Referências}

1. Edwards VJ, Anderson LA, Thompson WW, Deokar AJ. Mental health differences between men and women caregivers, BRFSS 2009. J Women Aging. 2017;29(5):385-91. https://doi.org/10.1080/08952841.2016.1223916

2. Glauber R. Gender Differences in Spousal Care Across the Later Life Course. Res Aging. 2017;39(8):934-59. https://doi.org/10.1177/0164027516644503

3. Minayo MCS. O envelhecimento da população brasileira e os desafios para o setor saúde. Cad Saúde Pública. 2012;28(2):208-9. https://doi.org/10.1590/S0102-311X2012000200001

4. Adelman RD, Tmanova LL, Delgado D, Dion S, Lachs MS. Caregiver burden: a clinical review. JAMA. 2014;311(10):1052-60. https://doi.org/10.1001/jama.2014.304

5. Ministério da Previdência e Assistência Social (BR). Idosos: Problemas e Cuidados Básicos. Brasília: Ministério da Previdência e Assistência Social; 1999.

6. Kasuya RT, Polgar-Bailey P, Takeuchi R. Caregiver burden and burnout. A guide for primary care physicians. Postgrad Med. 2000;108(7):119-23. https://doi.org/10.3810/pgm.2000.12.1324

7. Zarit SH, Reever KE, Bach-Peterson J. Relatives of the impaired elderly: correlates of feelings of burden. Gerontologist. 1980;20(6):649-55. https://doi.org/10.1093/geront/20.6.649

8. Cajado-Mejía R, Ruiz-Arias E. Influence of Gender and Care Strategy in Family Caregivers' Strain: A Cross-Sectional Study. J Nurs Scholarsh. 2016;48(6):587-97. https://doi.org/10.1111/jnu.12256

9. Scott J. Gênero: uma categoria útil de análise histórica. Educ Real. 1995;20(2):71-99.

10. Courtenay WH. Constructions of masculinity and their influence on men's well-being: a theory of gender and health. Soc Sci Med. 2000;50(10):1385-401. https://doi.org/10.1016/S0277-9536(99)00390-1

11. Calasanti T, Bowen ME. Spousal Caregiving and Crossing Gender Boundaries: Maintaining Gendered Identities. J Aging Stud. 2006;20(3):253-63. https://doi.org/10.1016/j.jaging.2005.08.001

12. Slack K, Fraser M. Husband, Partner, Dad, Son, Carer? A survey of the experiences and needs of male carers. London: Carers Trust; 2014.

13. Scazufca M. Brazilian version of the Burden Interview scale for the assessment of burden of care in careers of people with mental illnesses. Rev Bras Psiquiatr. 2002;24(1):12-7. https://doi.org/10.1590/S1516-44462002000100006

14. del Río-Lozano M, García-Calvente Mdel M, Marcos-Marcos J, Entrena-Durán F, Maroto-Navarro G. Gender identity in informal care: impact on health in Spanish caregivers. Qual Health Res. 2013;23(11):1506-20. https://doi.org/10.1177/1049732313507144

15. Walker AJ, Pratt CC, Shin H, Jones LL. Motives for parental caregiving and relationship quality. Fam Relat. 1990;39(1):51-6. https://doi.org/10.2307/584948

16. Sharma N, Chakrabarti S, Grover S. Gender differences in caregiving among family - caregivers of people with mental illnesses. World J Psychiatry. 2016;6(1):7-17. https://doi.org/10.5498/wjp.v6.i1.7

17. Camden A, Livingston G, Cooper C. Reasons why family members become carers and the outcome for the person with dementia: results from the CARD study. Int Psychogeriatr. 2011;23(9):1442-50. https://doi.org/10.1017/S1041610211001189

18. Schrank B, Ebert-Vogel A, Amering M, Masel EK, Neubauer M, Watzke H, et al. Gender differences in caregiver burden and its determinants in family members of terminally ill cancer patients. Psychooncology. 2016;25(7):808-14. https://doi.org/10.1002/pon.4005

19. Dupuis SL, Epp T, Smale B. Caregivers of Persons with Dementia: Roles, Experiences, Supports, and Coping - A Literature Review. Waterloo: MAREP; 2004.

20. Dwyer JW, Lee GR, Jankowski TB. Reciprocity, elder satisfaction, and caregiver stress and burden: The Exchange of aid in the family caregiving relationship. J Marriage Fam. 1994;56(1):35-43. https://doi.org/10.2307/352699 
21. Neufeld A, Harrison MJ. Reciprocity and social support in caregivers' relationships: Variations and consequences. Qual Health Res. 1995;5(3):348-65. https://doi.org/10.1177/104973239500500306

22. Lee Y, Tang F. More caregiving, less working: caregiving roles and gender difference. J Appl Gerontol. 2015;34(4):465-83. https://doi.org/10.1177/0733464813508649

23. Milligan C, Morbey H. Care, coping and identity: Older men's experiences of spousal care-giving. J Aging Stud. 2016;38:105-14. https://doi.org/10.1016/j.jaging.2016.05.002

24. Gunther H. Pesquisa Qualitativa Versus Pesquisa Quantitativa: Esta É a Questão? Psic Teor Pesq. 2006;22(2):201-10. https://doi.org/10.1590/S0102-37722006000200010

25. Konzen A, Giugliani C, Diercks MS, Vidal MA, Bertoni S. Cuidando de quem cuida: manual para quem cuida de uma pessoa que precisa de cuidados permanentes. Porto Alegre: Serviço de Saúde Comunitária do Grupo Hospitalar Conceição; 2003. 